Revista Iberoamericana, Vol. LXXIX, Núm. 242, Enero-Marzo 2013, 213-225

\title{
LA EMERGENCIA DEL SUJETO HOMOSEXUAL EN COSTA RICA: DOS TEXTOS PARADIGMÁTICOS
}

\author{
POR \\ URIEL QUESADA \\ Loyola University
}

\section{INTRODUCCIÓN}

En las historias de las minorías sexuales -así como en las de las literaturas- se pueden encontrar eventos y/o textos que marcan un antes y un después. En cuanto a las minorías, algunos de los sucesos más significativos van a girar en torno al tema de los derechos civiles. Los parteaguas literarios, por su parte, cambian de manera radical las formas de escritura, los contenidos o la visión de mundo. Algunas veces se puede hablar incluso de impactos sociales o culturales de largo plazo.

En el presente artículo quiero referirme a dos textos que pueden considerarse paradigmáticos en términos de la emergencia de una idea del sujeto homosexual en Costa Rica. El primer texto es una carta abierta publicada en el periódico costarricense La Nación el 5 de abril de 1987. Dicha carta pone en el centro de la esfera pública a la comunidad homosexual de Costa Rica, hasta entonces invisibilizada o identificada como portadora del SIDA, en aquel entonces una enfermedad mortal y a la vez social. El segundo texto es la novela de José Ricardo Chaves, Paisaje con tumbas pintadas en rosa, de 1998, libro en el que se hace una crónica de la vida de un grupo de homosexuales entre los años 1982 y 1989, y que ficcionaliza el medio en el que se da la carta abierta de 1987. En ambos textos hay propuestas implícitas y explícitas en cuanto a identidad, comunidad y noción de sujeto. Mi propósito es comparar ambos escritos -y en el caso de la carta sus antecedentes según los recoge el historiador Jacobo Schifter- en cuanto a sus estrategias para construir escrituralmente lo que sería un homosexual costarricense y sus relaciones con su medio social y político. 
II. LA CARTA DEL 5 DE ABRIL DE 1987, o LOS ESPACIOS PÚBLICOS

Hablando de las minorías y sus derechos, hay ciertos parteaguas que implican claramente una forma de empoderamiento, ${ }^{1}$ como lo sería la redada policial del 29 de junio de 1969 en el “Stonewall Inn”, un bar en Greenwich Village, Nueva York. Como se ha documentado ampliamente, esa noche la policía newyorkina llega por sorpresa y arresta a los cantineros y a algunos travestis que se hallan en el local. Al contrario de otras redadas, en esta ocasión la gente que se va aglomerando en la calle protesta, dando pie a varias jornadas de disturbios y posteriormente a lo que Jacobo Schifter en su libro La formación de una contracultura. Homosexualismo y Sida en Costa Rica, ha llamado “[e]l movimiento político gay más importante” (260), y cuya influencia se deja sentir en todo el mundo, incluso en una sociedad no muy abierta como la Costa Rica de los setenta y ochenta, y más específicamente en la forma de pensar de homosexuales jóvenes de entonces. "Las ideas de orgullo gay”, nos dice Schifter, “de rebelión contra los roles estereotipados, así como la imagen masculina, contribuyeron a la mentalidad de la nueva generación” (72). ${ }^{2}$

Muchísimo tiempo antes de lo sucedido en el Stonewall, el 17 de noviembre de 1901, otro acto de represión policial se constituye en un parteaguas social, político y cultural. Esta vez el escenario es la ciudad de México, y la redada se da en una casa particular, donde un grupo de cuarentiún hombres, varios vestidos de mujer, tiene una fiesta. Los asistentes a la fiesta son encarcelados, sometidos a humillación pública y enviados a exilio en Yucatán. Este grupo, conocido como los "Famosos 41", genera en su momento un escándalo con ribetes políticos ${ }^{3}$ y sociales que son recogidos en la prensa de entonces. ${ }^{4}$ Pocos años después el grupo será el tema de una novela, Los cuarenta y uno: novela crítico-social, publicada por Eduardo Castellón en 1906. Pero, más importante aún, Carlos Monsivaís afirma que la redada de setiembre de 1901

1 Uso la palabra empoderamiento como una derivación de empoderar. Alberto Bustos, profesor de la Universidad de Salamanca, afirma que el significado contemporáneo de dicha palabra proviene de "empower", cuyo origen a su vez son "los movimientos por los derechos civiles, que buscaban empoderar a sus seguidores, es decir, conquistar derechos y, con ellos, parcelas de poder para quienes estaban privados de ellos” (“Empoderar”). Por lo anterior me interesa el sentido político, social y cultural de la palabra.

2 Nótese, sin embargo, que Schifter limita la posible influencia de los movimientos gay norteamericanos a los homosexuales costarricenses, dejando por fuera la situación de las lesbianas, los y las bisexuales, así como otras minorías sexuales.

3 El escándalo pudo incluso implicar a la familia del dictador Porfirio Díaz, pues se supone que el cuadragésimo segundo participante de la fiesta era su yerno Ignacio de la Torre (Irwin 170).

4 Son significativas las caricaturas de José Guadalupe Posada sobre los "Famosos 41", en las que el artista se mofa de los arrestados y da una lección de control moral. Dichas caricaturas se pueden ver en el ensayo de Robert McKee Irwin, Edward J. McCaughan y Michelle Rocía Nasser titulado "Sexuality and Social Control in Mexico, 1901”, páginas 7, 8, 11 y 13.

Revista Iberoamericana, Vol. LXXIX, Núm. 242,
ISnero-Marzo 2154-4794 (Electrónico) 
"inventa la homosexualidad en México [...] modifica el sentido de esa colectividad en las tinieblas [...] y esta primera visibilidad es un paso definitivo" (citado en Irwin 16970). Para Monsivaís e Irwin los sucesos de 1901 dicen tanto sobre el grupo expuesto como sobre la sociedad que lo excluye, y aparejado al rechazo y la condena se puede leer entre líneas una fascinación por esas sexualidades alternativas, ocultas en la sombra, así como por la diversidad en sí. En este sentido, la memoria de lo ocurrido en 1901 no es una de represión "but of the ball scandalously joined together in the public imagination of transvestites, libertines, the sexually curious, and all kind of horny Mexicans of all social classes under the sign of the 41" (Irwin 183).

En Costa Rica hay por lo menos un hecho que hace saltar a la escena pública a la minoría homosexual como grupo de sujetos con identidades reales: en este caso será la publicación el 5 de abril de 1987 de una carta abierta en el periódico La Nación denunciando la represión del gobierno de Óscar Arias contra las personas homosexuales, a quienes se acosa como parte de una supuesta estrategia de prevención del SIDA. Dicha carta abierta aparece reproducida en $L a$ formación ${ }^{5}$ y podría decirse que cumple una doble función como texto de empoderamiento de las minorías sexuales en Costa Rica y como una forma de sexualización de la sociedad costarricense. ${ }^{6}$ Lo primero porque la carta surge en el contexto de una persecución sanitario-policiaca contra los homosexuales y lesbianas -aun cuando estas últimas no se consideraran en esa época como posibles portadoras del virus de inmunodeficiencia adquirida- y porque reclama respeto por derechos humanos y ciudadanos fundamentales. Lo segundo porque pone en el centro de la opinión pública la epidemia del SIDA como un hecho presente e innegable en la sociedad costarricense de entonces. El texto declara que hay minorías sexuales en una Costa Rica cuya construcción de la idea de nación estaba basada en una supuesta unidad étnica, religiosa y política, donde la heterosexualidad se da por sentada.

Al reconstruir los antecedentes de la carta publicada en abril de 1987, Schifter elabora dos vertientes. La primera se refiere a la propagación de información de corte homofóbico, la cual se inicia hacia 1985 cuando en la revista Rumbo se relaciona la propagación del SIDAcon “la 'perversidad' de los homosexuales” (101), mientras que en el vespertino La prensa libre se pide tanto "cuarentena para los gays" como "la clausura inmediata de todos los establecimientos gays" (101-2). Esta vertiente continúa por años, tanto por las publicaciones en los medios de prensa como por los propios mensajes que se emiten desde las oficinas de gobierno encargadas de combatir la epidemia.

5 Esta carta igualmente aparece en la novela de José Ricardo Chaves, Paisaje con tumbas pintadas en rosa, como se analizará posteriormente.

6 Robert Irwin cierra su ensayo sobre los "Famosos 41" con una aseveración categórica: "The ball not only Mexicanized homosexuality but also homosexualized Mexico. And Mexico would never be the same" (184).

ISSN 0034-9631 (Impreso) 
La segunda vertiente adquiere fuerza durantela administración deÓscar Arias, cuando se toman acciones abiertamente represivas contra los gays y lesbianas, específicamente al acosar a las personas en los centros de reunión por excelencia: los bares. Según Schifter, cerrar los bares de homosexuales no era permitido por la ley, por lo que el "Ministro de Salud, en asociación con su colega de Seguridad y el Viceministro de Gobernación Álvaro Ramos, optaron entonces por presionar a los bares y lograr su cierre por medio de la manera tradicional: las redadas” (109). Así las cosas, durante los primeros meses de 1987 se dieron operaciones policiales y arrestos. En algunos casos incluso hubo presencia de los medios de comunicación colectiva (110).

Schifter se preocupa por las conexiones entre homofobia y SIDA. En su reflexión, la epidemia incrementó los temores de la población por al menos las siguientes razones: "revelar el tamaño real de la comunidad gay" (113) y la "paranoia [provocada] por una comunidad invisible”(114), es decir, sin organizaciones abiertas ni una visibilidad política. Por esos temores, las personas empiezan a crear una imagen de los homosexuales basada en el deseo. Los gays, según lo relata Schifter, supuestamente se reúnen en los bares a practicar "orgías y bacanales” y “[a corromper a] menores” (112). Al contrario de cómo se construyó la imagen de los Famosos 41 en México-seres censurables por sus costumbres, pero a la vez tragicómicos y expuestos a la humillación pública- los homosexuales a los que Schifter se refiere son fundamentalmente corruptos, hipersexualizados y, lo más grave, invisibles, por lo cual su capacidad de contaminar a la sociedad (y el SIDA como símbolo de esa contaminación) sería no solamente muy grande sino efectiva y siniestra. En este contexto el gobierno halla una justificación para su política de redadas y arrestos arbitrarios que culminan en las primeras formas abiertas de organización homosexual en Costa Rica, y más específicamente en la carta del 5 de abril.

Los antecedentes de la carta podrían entenderse como un ejemplo de las relaciones entre sexualidad y el discurso médico-legal. Judith Butler, al hablardel SIDAy los discursos en torno a la epidemia, retoma a Foucault al afirmar que "[w]ithin the medico-juridical discourse that has emerged to manage and reproduce the epidemic of AIDS, the juridical and productive forms of power converge to effect a production of the homosexual as a bearer of death" (346). Ambas formas de poder son, sin embargo, de signo opuesto. La forma jurídica opera negativamente, es decir, normando, poniendo límites, restricciones y prohibiciones (Butler 345), mientras que la productiva elabora objetos e identidades y al mismo tiempo crea sus propios discursos de orden científico/regulatorio (345), que en el caso del SIDA sería el discurso médico que ampara las prohibiciones y las acciones represivas. En la superficie lo que se indica es la restricción a los homosexuales de reunirse, aunque más en lo profundo se encuentra la prohibición de ser, pues el homosexual, o la sospecha de serlo, lo convierte en un sujeto contaminante a partir de su identidad sexual; en el caso del paralelismo SIDA-homosexual, la contaminación implica la muerte.

\footnotetext{
Revista Iberoamericana, Vol. LXXIX, Núm. 242,
ISSN 0034-9631 (Impreso)
ISSN 2154-4794 (Electrónico)
} 
Al referirse a la represión, Foucault en su Historia de la sexualidad señala una característica esencial: "repression operate[s] as a sentence to disappear, but also as an injunction to silence, an affirmation of nonexistence, and, by implication, an admission that there was nothing to say about such things, nothing to see, and nothing to know" (4). En este sentido, el discurso médico-legal y las acciones policiacas posteriores son complementarios. El primero contribuye a crear la ficción que precede al homosexual, lo convierte no en portador sino en sinónimo de la enfermedad y, por ende, de muerte. El segundo hace desaparecer bajo un manto de silencio o sobrentendidos la supuesta fuente del mal.

A pesar de su poder como instrumento de cambio, ${ }^{7}$ la carta publicada en el periódico La Nación el domingo 5 de abril de 1987 no deja de ser un texto escrito en clave, que va creando laboriosamente sus puntos de transgresión con el poder. Es un texto que aborda su tema por los lados, claro en cuanto a ciertas acciones gubernamentales, aunque prefiere ser indirecto en cuanto a los sujetos afectados. Se utiliza una estrategia discursiva que omite palabras clave como homosexualidad, comunidad o minoría. Es un texto que procura denunciar sin ser totalmente explícito, casi un mensaje en clave a las autoridades políticas costarricenses. ${ }^{8}$

La carta abierta se dirige a los ministros de Salud, Seguridad Pública y Gobernación. El primer párrafo establece una relación causal entre miedo y represión como un hecho repetido históricamente, pero no lo hace entre poder y represión. Esta omisión nos invita a regresar a Foucault, específicamente a las conexiones entre represión, poder, conocimiento y sexualidad. Dice el filósofo francés en The History of Sexuality:

We are informed that if repression has indeed been the fundamental link between power, knowledge, and sexuality since the classical age, it stands to reason that we will not be able to free ourselves from it except at a considerable cost: nothing less than a transgression of laws, a lifting of prohibitions, an irruption of speech, a reinstating of pleasure within reality, and a whole new economy in the mechanisms of power will be required. (5)

En lo que se considera un estado de derecho como Costa Rica, la transgresión puede tomar ciertas formas dentro del aparato ideológico mismo. En el caso que nos interesa sería en primera instancia la denuncia pública, pero también la amenaza vedada de

7 Aparte del fin de las redadas, en los meses posteriores a esta publicación, el SIDA entraría al medio costarricense como tema abierto de discusión (Schifter 115), se detendría cualquier intento de exámenes médicos forzosos a los empleados públicos (ya planteados como forma de prevención), y sería un dinamizador de las organizaciones gay en Costa Rica (263).

8 Es importante tener en cuenta que 1987 es un año fundamental para la administración Arias y para el Presidente Óscar Arias en particular. Por sus esfuerzos para lograr el cese de los conflictos bélicos en América Central es honrado con el Premio Nobel de la Paz.

ISSN 0034-9631 (Impreso) 
atacar a la estructura de poder en algo sensible: el prestigio de mantener un diálogo social basado en la defensa de los derechos humanos.

Seguidamente la carta abierta pasa a enumerar tres formas en que se manifiesta la represión, esto es contra "las ideas, la libertad y la dignidad de los seres humanos" (Schifter 291). Luego menciona la intolerancia y, en una progresión, el fanatismo, la pérdida de derechos y la persecución "por razones de raza, de religión, de sexo o de ideología” (291). Si bien, en esta última mención se encuentra implícito el tema de las minorías, no se habla ni se hablará en la carta de grupos minoritarios por orientación sexual.

Los homosexuales, por quienes se escribe este texto, aparecen por omisión. La palabra que los substituye y, en cierto modo representa, es SIDA. En el párrafo siguiente se dice que el SIDA “asusta hoy a todos los habitantes del planeta” (Schifter 291), por lo que, siguiendo la lógica establecida al principio del texto, el SIDA está detrás del miedo que lleva a la represión. En cierta manera se justifica la represión, por cuanto hay un hecho concreto, la epidemia, que la desencadena. No obstante, como ya se ha discutido, queda implícita la equivalencia enfermedad-homosexualidad-muerte.

Mencionado el mal, se establece un nexo con Costa Rica, con los casos aparecidos y las campañas oficiales para evitar que se propague la enfermedad. "Empero, ni el temor de todos ni la justa preocupación de las autoridades públicas, autoriza ni legitima las medidas represivas, inconstitucionales y violatorias de los más elementales derechos humanos" (Schifter 291). He aquí dos estrategias discursivas esenciales. Se vuelve a mencionar el temor público -el de todos- junto al oficial -la preocupación, pero esta vez se cuestiona la represión en términos concretos. Se van a mencionar de inmediato "las redadas nocturnas, indiscriminadas, vejatorias e infamantes, [que] no constituyen ninguna medida preventiva del SIDA y sí lesionan garantías constitucionales básicas de los costarricenses" (291). La conexión es sutil pero clara para quien haya estado al tanto de la situación de acoso y persecución. Quien no sepa lo que ha pasado en los meses previos, probablemente no podrá entender los detalles específicos del reclamo.

Los signatarios toman una posición aún más firme al demandar que "[l]as otras medidas, las que discriminan, reprimen policialmente y lesionan gravemente la dignidad de las personas, deben ser eliminadas de inmediato" (Schifter 292). ${ }^{9} \mathrm{Y}$ aunque no se "[avale] la vida privada de nadie, tampoco [se debe juzgar]" (292) pues el imperativo democrático es la igualad ante la ley. En este momento el sujeto homosexual implícito en la sigla SIDA pasa a ser un sujeto de derecho, y es así como la carta se convierte en un texto radical a pesar de los circunloquios, las omisiones y los paralelismos entre sujeto y muerte. Hay un reconocimiento del derecho del sujeto homosexual e ignorarlo ya no

9 Lo que debería hacerse, según los firmantes, es fomentar campañas educativas y la prevención, al alertar a la población sobre los riesgos inherentes a la epidemia del SIDA.

ISSN 0034-9631 (Impreso) 
es posible: "Empezar a distinguir a los costarricenses con etiquetas infamantes, atenta contra todas nuestras tradiciones de civismo y democracia y es una peligrosa puerta abierta a la arbitrariedad y al terrorismo de Estado" (292). Esas "etiquetas infamantes" no se aclaran nunca, son uno de varios sobreentendidos, pero la carga del párrafo se centra en la acusación de terrorismo de estado.

Schifter menciona en su libro algunas consecuencias directas, de corto plazo, de la carta del 5 de abril. La principal es que suspendieron las redadas ya para mediados de 1987 (116) $\cdot{ }^{10}$ Otra fue la convergencia de representantes de otras minorías, como la afro-costarricense y la judía, así como de la izquierda, en torno al problema del SIDA y la represión gubernamental (115). Ya en el mediano plazo “[...] la situación creada por la epidemia del Sida terminaría con el anonimato gay. La enfermedad se presentó como un "acelerador" para la organización” (263). En general, se puede afirmar que la comunidad entra en un proceso de empoderamiento, y que la carta del 5 de abril saca a la luz a un sector de la población urbana costarricense silenciada hasta entonces. De esta forma el discurso sobre la homosexualidad entra al pleno de la sociedad. Si en los años inmediatos anteriores lo hizo en función de la enfermedad y la contaminación tanto física como moral, ahora lo hace desde la perspectiva de los derechos individuales y la tradición de respeto a los demás y a la ley. Este equilibrio es, sin embargo, momentáneo, pues el ligamen homosexual-SIDA sigue alimentando su propia ficción y mantiene a los miembros de la comunidad LGBT bajo la perspectiva de lo que Butler ha llamado una morfología heterosexual, en la cual aquello que es "improperly sexed" (354) no es necesariamente humano y por tanto no es objeto de derecho. Así como en esa morfología se crea un efecto de "borrado" de lo femenino -pues la definición de sexo es masculina y lo femenino existe como espejo de esa definición, no como autonomía (354)-de igual forma la homosexualidad sale de la normativa heterosexual masculina y carece, por lo tanto, de ese rasgo identitario inherente que la define como característica del ser humano. Sería un ser otro, por una parte carente de derechos, pero a la vez de posible corrección, si es capaz de recuperar sus rasgos masculinos.

Podríamos entonces concluir que un acto de visibilidad radical como la carta abierta del 5 de abril de 1987 tiene un efecto en dos vías: de la minoría a la sociedad en sentido amplio y viceversa.

\section{Paisaje con tumbas Pintadas en ROSA, De José Ricardo ChaVes, o los espacios PRivados}

Aunque aún es prematuro hablar de una literatura gay costarricense o centroamericana -falta, por ejemplo, la labor de examinar exhaustivamente el corpus de obras donde

${ }^{10}$ Alexánder Obando, sin embargo, habla de una nueva época de represión en la década siguiente, durante la administración Figueres Olsen (1994-1998) (XVIII).

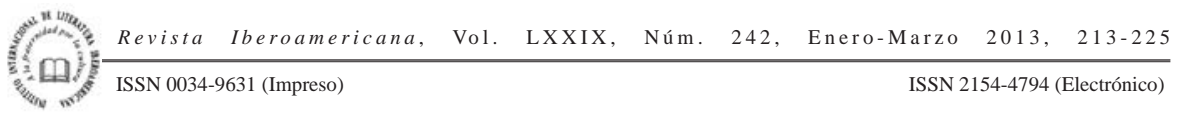


aparezcan personajes LGBT-es evidente que la presencia y el tratamiento de personajes LGBT ha ido cambiando a partir de los años ochenta. ${ }^{11}$ Uno de los autores pioneros es José Ricardo Chaves, quien publica su primer libro, La mujer oculta, en 1984. Ya en algunos cuentos de esta colección Chaves empieza a indagar las relaciones entre espacio y homosexualidad. En las narraciones de Chaves el homosexual halla lugares significativos en términos de sus conflictos y sus goces. El (re)conocimiento de sí mismo y de su comunidad se constituye a partir del entorno -urbano principalmente-, la memoria -metaforizada por la niñez- y el deseo. Hablamos entonces de la casa, el barrio, la finca, los bares... o de modo más sobresaliente: la ciudad de San José.

Esta exploración alcanza su punto más alto en la novela Paisaje con tumbas pintadas en rosa, de 1998. En esta obra la presencia de los homosexuales recodifica la noción de espacio. El lector encuentra individuos que se apropian de territorios no designados previamente por códigos heteronormativos en los cuales los sujetos se han de reconocer e interactúan en función de una dinámica identitaria y donde el deseo cumple un rol central. En estas apropiaciones hay una propuesta de comunidad homosexual, la cual se inserta en el panorama histórico de la década de los ochenta en Costa Rica y, más concretamente, en el contexto de la aparición de la pandemia del SIDA.

La obra está estructurada sobre tres ejes. Hay una narración principal, la de Óscar, un joven estudiante universitario de clase media. De él conocemos su círculo de amigos y la relación afectivo-sexual que entabla con un profesor de historia, Mario. El segundo eje lo componen cartas que una serie de personajes -en su mayoría anónimos o vagamente identificados-se intercambian. Mientras la narración principal se ocupa de la vida privada de los personajes centrales, las cartas se constituyen en una crónica de eventos en la esfera pública, poniendo la historia privada en un contexto social amplio. Por las cartas el lector llega a saber que la novela comienza alrededor de julio de 1982 y termina hacia principios de 1989. Las cartas también establecen un puente entre los homosexuales que viven en Costa Rica y sus pares que se hallan en el extranjero. Entre ellos hay un intercambio constante de información sobre amigos comunes, acontecimientos en Costa Rica y también reflexiones sobre lo que ocurre en el mundo. El tercer eje son recortes de periódico. ${ }^{12}$ Los recortes o breves notas de prensa dan cuenta de las reacciones a escala mundial cuando el SIDA aparece, principalmente en cuanto a la represión contra los homosexuales y las primeras manifestaciones de resistencia. Los tres ejes confluyen en una unidad, de tal suerte que la crónica de Óscar y sus amigos es a la vez la crónica de

${ }^{11}$ Un parámetro para sostener esta afirmación sería la antología La gruta y el arcoiris. El libro incluye textos de doce autores, con solamente dos textos correspondientes a periodos anteriores a 1980.

12 Sergio Coto Rivel se ha dedicado a documentar las fuentes del discurso médico-jurídico en torno al SIDA y la homosexualidad en los ochentas como parte de su análisis de la novela Paisaje con tumbas pintadas en rosa, de José Ricardo Chaves. Coto Rivel hace una indagación sobre las informaciones periodísticas presentes como intertextos en la novela.

$\begin{array}{llllll}\text { ISSN 2154-4794 (Electrónico) } & \text { Revista Iberoamericana, Vol. LXXIX, Núm. 242, Enero-Marzo 2013, }\end{array}$ 
un país al borde un cambio social y de una cultura en los inicios de la pandemia. Como parte del tercer eje de la narración aparece la carta abierta del 5 de abril (Chaves 149-50).

Jacobo Schifter, en su ya citado La formación de una contracultura, concluye que "[...] [n]o existía una comunidad gay en Costa Rica antes del Sida. Para que ello así hubiera sido, se necesitaría un grupo de personas que se autocalificaran como gays y que tuvieran una subcultura e instituciones propias” (106). El mismo Schifter, sin embargo, previo a esa conclusión describe diversas formas de relación homosexual que existían en el país desde los años cincuenta, en torno a bares y fiestas en casas y en fincas particulares. Para Schifter, una comunidad homosexual sólo sería posible en el contexto de un determinado desarrollo urbano, un espacio que incluyera la formación de guetos y la aparición de asociaciones de apoyo. Hay en sus ideas dos elementos implícitos: por una parte un modelo de comunidad, el norteamericano, y por otra, la expectativa de una incidencia social y política directa.

Paul Kutsche, en su ensayo "Two Truths about Costa Rica”, llama al modelo de Schifter "the opression model". En este modelo los homosexuales se encuentran sujetos a condiciones adversas (opresión económica, roles de género basados en una estructura patriarcal que equipara al homosexual con la mujer y que define a ambos humanos grupos como inmaduros y débiles) que los colocan "[at] the margins of society if they cannot hide their identity" (123). Kutsche elabora una perspectiva más conciliatoria de la visibilidad y de las formas de interacción de los homosexuales en Costa Rica en las décadas de los setenta y ochenta a la que llama “contractual model”. Sus rasgos básicos serían una base identitaria amplia -pues incluye a hombres que tienen sexo con otros hombres pero no se consideran gays- y un modelo cultural que favorece la expresión sexual. "The most important part of the sexual structure that I see", apunta Kutsche, "is that sex between men is two-layered. The public layer is homophobic, the private layer is quietly permissive and at times even favorable to male homosexuality" (111-12). Refiriéndose a dichos periodos Kutsche afirma que el costarricense en general no se identificaba a sí mismo como gay sino como una persona de ambiente o entendido. Ser de ambiente, según Kutsche, incluiría tanto a los que actuaban de manera masculina hasta la llamada loca (112) o el playo costarricense. Por su parte, para Robert Richmond Ellis, el ambiente "can be experienced only collectively, even if this collectivity is initially private” (4). Es una forma social, inclusiva y mudable, más cercana a lo performativo que a una identidad en sentido estricto o a un conjunto de organizaciones más o menos estables como apunta Schifter.

En "El mundo soslayado", un ensayo introductorio a la autobiografía de Salvador Novo, La estatua de sal, Carlos Monsiváis se refiere con libertad al gueto en las épocas que describe Novo. En un momento, Monsiváis concluye: "En La estatua de sal, un tema básico es el ingreso al ghetto homosexual, el entrar al Ambiente" (39, énfasis de Monsiváis). De esta manera establece una equivalencia entre gueto y ambiente, por lo

\footnotetext{
Revista Iberoamericana, Vol. LXXIX, Núm. 242,
ISSN 0034-9631 (Impreso)
} 
tanto habla de un espacio que incluye lo geográfico pero como otro componente en un conjunto más complejo, siempre dominado por la presencia del homosexual. Alberto Mira considera el gueto tanto "un estado psicológico (es decir, una serie de códigos semisecretos o de redes de influencia) como un entorno físico en el que se [puede] expresar la identidad sexual” (355). Este autor prefiere hablar de una topografía homosexual, término más abarcador que ambiente o gueto, y que incluye "una serie heterogénea de lugares de encuentro" (262). Mira identifica cuatro tipos de espacio: los escenarios de carácter social -bares o clubs-, las instituciones sexuales -saunas o prostíbulos-, los puntos de convergencia o entornos -el teatro, el clero, las organizaciones deportivas-, y los puntos de homosexualidad situacional -prisiones, el ejército o los internados-.

Si la presencia activa o performativa del homosexual define el ambiente, Paisaje con tumbas pintadas en rosa es una novela sobre el ambiente y por tanto sobre una forma de comunidad homosexual, en este caso la de Costa Rica en la década de los ochenta. Su radio excede la territorialidad impuesta por la visión heteronormativa ${ }^{13}$ para extenderse por amplias zonas de la ciudad de San José. El ambiente, a partir de la exclusión que implica en términos del proyecto nacional, tiende a una forma comunitaria sin fronteras expresamente demarcadas o, como dice el narrador de Paisaje: "Lo mismo en San José que en San Francisco. Lo mismo en París que en México: un bar gay es lo mismo en todos lados. Eso piensa Mario que acaba de ponerse a bailar con uno de sus amigos. No cosmopolitismo, sino supranacionalidad" (128). El bar de ambiente aparece como el eje de las relaciones homosexuales, aunque en Paisaje hay cabida a otros espacios: sodas y bares que acogen ampliamente la disidencia, como, por ejemplo, Chelles o La Copucha; las cafeterías universitarias, los parques y plazas de ligue del centro de San José; incluso se vuelve terreno para el flirteo y la conquista la multitud reunida para ver desfilar al Papa Juan Pablo II en Costa Rica. El espacio urbano es constantemente recodificado por la presencia y la interacción de los homosexuales, por sus búsquedas afectivas, eróticas e identitarias.

En Paisaje encontramos todas las categorías de espacio que apunta Alberto Mira, con la excepción del espacio situacional. La obra propone una visión distinta de San José en el sentido de que la ciudad es territorio gay, aún con sus exclusiones y silencios. Hay en este sentido una escena reveladora que anuncia la llegada del SIDA a Costa Rica. Óscar y su amigo Javier contemplan la ciudad de San José desde lo alto de una montaña. El narrador describe "cielos y pastos en colinas que ascienden. En la sima están las “[...] ciud/hades [sic], los edificios pululantes y el gentío. Hacia la cima, ellos”

${ }^{13}$ Véase por ejemplo el gay-to [sic] descrito en la novela de Carlos Cortés, Cruz de olvido (1999), o la zona al sur de San José en la novela Mariposas negras para un asesino, de Jorge Méndez Librick (2004). En ambos libros hay sectores urbanos específicos encapsulados por ciertas calles, dentro de los cuales junto a la homosexualidad impera la sordidez y el crimen.

ISSN 0034-9631 (Impreso) 
(93). Desde la óptica del narrador, la muerte es la misma ciudad y el ángel negro sería la epidemia del SIDA. De hecho, la enfermedad se representa como un "gigantesco hombre de humo" que al azar "[saca] hombres de lo hondo del valle" (97), es decir, de la ciudad donde la comunidad se asienta. El homosexual como sujeto no es portador de muerte sino su víctima, y aparecen fuerzas externas e incontrolables que son las portadoras reales de lo fatal.

Apartir de este momento la relación homosexual-espacio empieza a cambiar. Cuando a Javier se le diagnostica seropositivo hace un recorrido por el céntrico Paseo Colón, "que siempre le había resultado tan familiar, tan suyo, cuyas casas, oficinas, hospitales y restaurantes sabía casi de memoria, pero que estos momentos perdían peso, perdían familiaridad, para tornarse en escenario de un mundo en desintegración” (131). La posibilidad de la muerte pone en crisis el modelo vigente de comunidad, incluyendo el sentido de pertenencia. También ciertas formas de equilibrio se han roto, en particular el respeto a los territorios abiertamente gay, pues el mundo heterosexual empieza a invadir los lugares de ambiente. El silencio y la tolerancia que estaban implícitos hasta entonces dan paso a formas de violencia, a represión y atropello de derechos humanos, incluyendo crímenes de odio. La cruzada sanitaria que impulsa el gobierno se entiende a la vez como una cruzada de doble moral, y por ello la inclusión de la carta del 5 de abril puede considerarse como una respuesta desde la comunidad y sus aliados. A partir de esos hechos debe hablarse no de la formación de una comunidad sino de su reinvención, la cual se va a expresar por la acción pública directa, la organización y, desde el punto de vista del narrador en Paisajes, el commitment, el compromiso. El personaje principal de la obra, Óscar, optará sin embargo por una opción más radical: el exilio. Para entonces, ha muerto quien ha marcado su primera relación amorosa intensa, la ciudad ordenada ha revelado sus mecanismos de control y represión, y el mismo Óscar tiene ahora la posibilidad económica de dar el salto hacia otro nivel de la comunidad, ése que en los ochenta llegaba por las cartas y las notas de prensa. En efecto, sale a Nueva York, Roma y de ahí a México, donde una relación afectiva definirá nuevos lazos con el territorio.

En conclusión, ambos textos analizados abordan situaciones similares-la comunidad gay costarricense de los ochenta, la epidemia del SIDA, la represión-y nos presentan un sujeto homosexual que hasta entonces se hallaba totalmente marginalizado. Mientras que el primero de dichos textos, la carta abierta del 5 de abril de 1987, tiene consecuencias político-sociales inmediatas y visibiliza a la comunidad, no escapa de ciertos códigos represivos como lo sería las omisiones de palabras clave o la equivalencia entre SIDA y homosexual. El segundo texto, la novela Paisaje con tumbas pintadas en rosa, de José Ricardo Chaves, ficcionaliza los antecedentes de la carta, aunque su interés radica en hacer una crónica de la vida homosexual urbana en el San José de la época. Al incluir la carta del 5 de abril como un elemento más en la ficción da a los lectores una perspectiva desde adentro, desde el grupo minoritario, y construye una representación positiva del

\footnotetext{
Revista Iberoamericana, Vol. LXXIX, Núm. 242,
ISSN 0034-9631 (Impreso)
} 
homosexual como sujeto de derecho y como ser humano en sí mismo, inmerso en una dinámica social, afectiva y erótica.

La novela también muestra una forma concreta y posible de comunidad -ausente en la carta abierta y sus antecedentes- renunciando a un modelo de aislamiento o de gueto por una red de personas que se identifican, interactúan y apoyan.

\section{BiBLIOGRAFÍA}

Bustos, Alberto. “Empoderar”. Blog de Lengua Española. 12 junio 2008. <http://blog. lengua-e.com/2008/empoderar/>. 16 dic. 2009.

Butler, Judith. "Sexual Inversions". Discourses of Sexuality from Aristotle to AIDS. Donna C. Stanton, ed. Ann Arbor: U of Michigan P, 1992. 344-61.

Chaves, José Ricardo. La mujer oculta. San José: Costa Rica, 1984. Paisaje con tumbas pintadas en rosa. Heredia: Universidad Nacional, 1998.

Coto Rivel, Sergio. "Una década perdida, noticias del miedo en Paisaje con tumbas pintadas en rosa de José Ricardo Chaves”. Istmo 19 (2009). < http://collaborations. denison.edu/istmo/n19/articulos/3-coto_sergio_form.pdf>. 20 dic. 2009.

Ellis, Robert Richmond. Introducción. Reading and Writing the Ambiente. Queer Sexualities in Latino, Latin American, and Spanish Culture. Susana ChávezSilverman y Librada Hernández, eds. Madison: U of Wisconsin P, 2000. 3-18.

"Empower". 2da acepción. American Heritage College Thesaurus. Boston: Houghton Miflin, 2004.

“Empowerment”. 3ra acepción. Merriam-Webster Dictionary. <http://www.merriamwebster.com/dictionary/empowerment>. 16 dic. 2009.

Foucault, Michel. The History of Sexuality. An Introduction. Volume I. Nueva York: Vintage Books, 1990.

Irwin, Robert McKee. "The Centenary of the Famous 41”. The Famous 41. Sexuality and Social Control in Mexico, 1901. Robert McKee Irwin, Edward J. McCaughan y Michelle Rocío Nasser, eds. Nueva York: Palgrave, 2003. 169-89.

Irwin, Robert McKee, Edward J. McCaughan y Michelle Rocío Nasser. "Introduction: Sexuality and Social Control in Mexico, 1901”. The Famous 41. Sexuality and Social Control in Mexico, 1901. Irwin, McCaughan y Nasser, eds. Nueva York: Palgrave, 2003. 1-18.

Kutsche, Paul. "Two Truths about Costa Rica”. Latin American Male Homosexualities. Stephen O. Murray, ed. Albuquerque: U of New Mexico P, 1995. 111-37.

Mira, Alberto. Para entendernos. Diccionario de cultura homosexual, gay y lésbica. Barcelona: Tempestad, 1999.

Monsivaís, Carlos. "El mundo soslayado”. La estatua de sal. Salvador Novo. México: Consejo Nacional para la Cultura y las Artes, 1998. 1-41.

$\begin{array}{llllll}\text { ISSN 2154-4794 (Electrónico) } & \text { Revista Iberoamericana, Vol. LXXIX, Núm. 242, Enero-Marzo 2013, }\end{array}$ 
Obando, Alexánder. Prólogo. La gruta y el arcoiris. Antología de narrativa gay/lésbica costarricense. Alexánder Obando, ed. San José: Editorial de Costa Rica, 2008. III-XLII.

Schifter, Jacobo. La formación de una contracultura. Homosexualismo y SIDA en Costa Rica. San José: Guayacán,1989.

$\begin{array}{lllll}\text { Revista Iberoamericana, Vol. LXXIX, Núm. 242, Enero-Marzo 2013, 213-225 } & \text { ISSN 2154-4794 (Electrónico) }\end{array}$ 
